ture", by B. C. Vickery, which is of some scientific interest. Mr. Vickery summarizes, with a useful bibliography and some account of the mothods of study which have been used, the kinds of information sought by scientists, the extent and nature of their reading and the forms of document used. The survey shows that the quantitative contribution to the reading and learning of scientists made by formal bibliographical tools is equalled by the contributions of present-day scanning, cited references, and of oral recommendations. Mr. Vickery recognizes, too, the importance of oral communication, but he indicates rather than discusses the implications of such conclusions as are reached, and emphasizes the need for further research on ways of improving the present position. A second article in the same issue, by W. L. Emerson, discusses an approach to book selection in the sciences for public libraries.

\section{The Geological Society of India}

IN January 1959 the Geological Society of India was founded to promote the advancement of geological study and research throughout all India and to co-operate with similar institutions in other lands. One of the aims of the new Society is the publication of a journal, and of monographs and memoirs, dealing with special problems of Indian geology; and the first two volumes from this source have now appeared (Journal of the Geological Society of India, Vol. 1, 1959. Pp. $166+6$ plates. Bangalore: Goological Society of India, 1959. Rs. 15). This periodical embraces the whole field of geological science, the first two issues comprising 25 short papers mostly of a high standard with many of world-wide interest. In the first presidential address, Dr. D. N. Wadia deals with "The Evolution of the Desert Belt of Asia", delineating briefly, but brilliantly, the overall geological problems of the vast arid tracts which stretch from the Red Sea to Mongolia. Within the past decade the number of geologists in India has increased greatly, and the potential importance of the geological contribution to national development there is becoming widely recognized. The Geological Society of India is likely to play a major part in maintaining the long-established tradition of sound work, and its publications will be warmly welcomed in geological libraries throughout the world.

\section{Atomic Energy-levels in Crystals}

Atomic Energy Levels in Crystals, by J. L. Prather, National Bureau of Standards Monograph No. 19, which was issued on February 24 , presents a theoretical study of the sharp-line absorption spectra of crystals (National Bureau of Standards. Monograph No. 19. Pp. iv +84 . Washington, D.C.: Government Printing Office, 1961. 60 cents). It is largely an exhaustive collection of the results of group theory that apply to the theory of atomic energy. levels in crystals, together with a detailed develop. ment of the general theory to permit application of the results to experimental problems. The discrete energy-levels observed within certain crystals are treated as being due to perturbations of the energylevels of the free ion by an electrostatic field arising from the crystal lattice. The analytic procedures for determining the field from the charge configuration are given, and the resulting fields are classified according to their symmetry. After a general survey of group-theoretical ideas, the applicable groups are analysed in detail, and characters appropriate for both integral and half-integral angular momenta of the free ion are tabulated. The monograph contains eighteen tables and an extensive bibliography. The review on the general subject entitled "Group Theory and Crystal Field Theory", written by Dr. C. M. Herzfeld in collaboration with Prof. P. H. Meijer, which is to be published shortly by the National Bureau of Standards, Washington, treats the subject from the more abstract point of view of modern algebra and concentrates on the fundamental aspects of the theory, and will be a complementary work to that of the monograph.

\section{Travelling-wave Tubes}

THe travelling-wave tube is rapidly becoming established as an essential component in microwave links used for civil communications. It is a microwave amplifier that operates on the principle of a continuous interaction between the input signal and an electron beam as they travel together through the tube, and is particularly suitable for operation at frequencies greater than 1,000 Mc./s. It has a broad band-width and high gain. An outline of the construction, characteristics, operation and typical applications of the travelling-wave tube is given in the illustrated booklet, Travelling Wave Tubes : Their Characteristics and Operation, issued by the English Electric Valve Co., Ltd. (Pp. 40. Chelmsford: English Electric Valve Co., Ltd., 1961). Following a general description of the tube and its associated circuitry, explained by means of a simplified schematic diagram, the booklet discusses the various types of travelling-wave tubes now commercially available. They can be classified according to their functions into three major groups-power tubes, medium power or intermediate tubes, and low-noise tubes-and these can be subdivided, according to the type of $r f$ connexion used, into either wave-guide or coaxial mounting types. All the travelling-wave tubes produced by the English Electric Valve Co., Ltd., require a longitudinal magnetic focusing field for maintaining the electron stream in a concentrated beam over the length of the tube. One section of the booklet deals with focus systems for the tubes, and another with the procedure to be followed in the installation of a tube into a circuit. In addition to the use of travelling-wave tubes as microwave amplifiers in both terminal and repeater stations in communication links, the low-noise tube is being increasingly used in the input stage of radar receivers as a pre-amplifier to the crystal mixer, and travelling. wave tubes may also be used as amplitude or phase modulators, frequency changers, stable oscillators or reflex amplifiers. The booklet is intended as an introduction only to the subject of travelling-wave amplification, and for those who want a more detailed. treatment a bibliography of sixteen references is provided. The abridged data listed refer to the English Electric tubes and solenoids.

\section{Lalor Grants and Awards for 1961}

THE Lalor Foundation has announced the programme of awards for 1962, which it is offering for support of research on the fundamental biochemical and physiological mechanisms concerned with fertility and the early stages of reproduction in various forms of life. The objectives are to further the knowledge and understanding of the basic phenomena involved and to extend and develop the possibilities for effective regulation and control. The awards are open 